\title{
Optimal Generalized Predictive Functional Control with Applications to Extended PID Control for Quadruple Tank
}

\author{
Sultan Alotaibi, Michael Grimble \\ Department of Electronic \& Electrical Engineering \\ University of Strathclyde, Glasgow, UK \\ Email: sultan.m.alotaibi@strath.ac.uk; m.j.grimble@strath.ac.uk
}

\begin{abstract}
Algorithm of optimal Generalized Predictive Functional Control is presented towards the control problem of linear discrete-time state-space multivariable systems. A quadruple tank system is simulated to demonstrate typical results. The proposed controller has been structured in a reduced order arrangement including a set of specified linear transfer-functions along with gains that are found to minimize a GPC cost-index. This approach enables a range of classical controller structures to be used in the feedback loop such as extended PI, PID or Lead-lag or a more general transfer function form. A MATLAB simulation was used to test the performance of the restricted structure controller for regulation and tracking problems and was compared against a classical PI Controller.
\end{abstract}

Keywords- multivariable systems, predictive PID, generalized predictive control, predictive functional control

\section{INTRODUCTION}

It is well known that PID control is very popular in industry and is used very effectively in most industrial areas, but if the dynamics of systems are relatively complicated, it is possible that PID control will not deliver decent performance. An optimal controller with higher order may therefore, be essential to also deal with multivariable system dynamics [1]. And it would be therefore, seem realistic to extend the concept of PID control by expanding the controller to provide more design freedom. One idea may be to add some functions which can be chosen to in some way to cover the range of frequency responses that may be needed. When discussing the use of functions, the idea of Jacques Richalet regarding Predictive Functional Control (PFC) in projecting the manipulated variable or control signal onto a functional basis, do of course come to mind [2]-[4].

In this paper, the controller structure will be therefore, in the usual feedback form, but the controller structure will be defined as a set of frequency-sensitive functions. These are multiplied by gains which will be determined in same principal of Model Based Predictive Control (MBPC) through minimizing a Generalized Predictive Control (GPC) style cost-function that could for example correspond with PI or PID type terms. Of course, many efforts have been introduced to bring together the aids of PID with predictive control. Predictive PID controller was introduced in [5], with characteristics of MBPC. It was shown that the controller turns down to the equivalent structure as a PI or PID controller for $1^{\text {st }}$ and $2^{\text {nd }}$ order systems, correspondingly. In [6], a predictive PID control algorithm was developed with performance comparable to that of a GPC controller with an aim to introduce an online optimization technique, to tune PID controllers, which can function as PID or as multivariable GPC controller. The Generalized Predictive Functional Control (GPFC) approach introduced here towards highly interactive multivariable Quadruple Tank Process (QTP), can be related to these results in a limiting case when the functions are related to PI or PID inspired functions. While in this approach, a range of classical controller structures can be used in the feedback loop such as extended PI, PID or even more general transfer function form. Therefore, the controller is of restricted structure form and the solution is not the same as published by Richalet and others because it has the advantage of providing the natural robustness of low order controllers and performance related to predictive control algorithms. One of the important assumptions, in the solution was that the system experienced enough excitation so that the rank condition on a matrix whose inverse was required was satisfied [6]. This is not an assumption that is needed in the following, except in the very special situation when the weighting on the controller gains tend to zero.

This paper has been organized as follows: the linear plant, disturbance models and models for prediction are described in Section II. The structure of the restricted structure controller is introduced in section III. The cost minimization problem and the solution of the GPFC problem is considered in IV. The performance analysis and simulation comparison presented in section V. Finally, conclusions are summarized in section VI.

\section{SYSTEM EXPLANATION}

The complete system, is depicted in Fig. 1 and consist of, a linear plant model along with reference, disturbance sources and measurement noise [7]. The disturbance signal is assumed to be represented by LTI model excited via zero mean white noise and a measured output disturbance $d(t)$. The reference signal $r(t)$, is also expected to be deterministic. The measurement noise $v(t)$, is expected to retain a fixed covariance matrix and the noise signal $\zeta(t)$ has an identity covariance matrix. The plant subsystem in (1), could have a general linear operator form whereas just the input-output model is available [8].

$$
\left(W_{1} u\right)(t)=z^{-k}\left(W_{1 k} u\right)(t)
$$




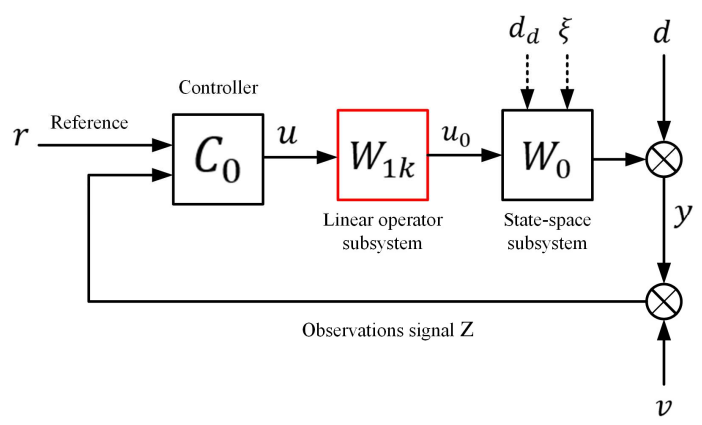

Figure 1. GPFC for Unstructured Linear \& State Space Subsystems

and $z^{-k} I$ represents a diagonal matrix contains the delay parts at output signal channels thru $k>0$. The output of the linear subsystem $W_{l k}$ is represented as $u_{0}(t)=\left(W_{l k} u\right)(t)$. The subsystem $W_{l}$ is expected to be stable but, the second linear subsystem denoted $W_{0}=z^{-k} W_{0 k}$ established in comprehensive way in the next, can include unstable modes. If there is no need for the first linear subsystem, then $\mathrm{W}_{1 \mathrm{k}}=I$. This formalization of different delays in in various signal channels has been introduced in a simple way in [9]. The weighted error can contain any stable dynamic cost function weighting such $e_{p}(t)=P_{c}\left(z^{-1}\right) e(t)$. Noting that, the linear subsystem models that describe the augmented system, will not be studied entirely here since it is already discussed in [7], [8].

Considering only this state space subsystem and linked to the system measured outputs. This model is augmented to include a defined model of the disturbance and error cost weighting operator $P_{c}\left(z^{-1}\right)$ and to be stabilizable and detectable and denoted in state space:

$$
x(t+1)=A_{t} x(t)+B_{t} u_{0}(t-k)+D_{t} \xi(t)+d_{d}(t)
$$

\section{Augmented System State-Space Model}

$$
\begin{gathered}
{\left[\begin{array}{c}
x_{0}(t+1) \\
x_{d}(t+1) \\
x_{p}(t+1) \\
x_{r}(t+1)
\end{array}\right]=\left[\begin{array}{cccc}
A_{0} & 0 & 0 & 0 \\
0 & A_{d} & 0 & 0 \\
-B_{p} C_{0} & -B_{p} C_{d} & A_{p} & 0 \\
0 & 0 & 0 & A_{r}
\end{array}\right]\left[\begin{array}{l}
x_{0}(t) \\
x_{d}(t) \\
x_{p}(t) \\
x_{r}(t)
\end{array}\right]+\left[\begin{array}{c}
B_{0} \\
0 \\
-B_{p} E_{0} \\
B_{r}
\end{array}\right] u_{0}(t-k)} \\
+\left[\begin{array}{cc}
D_{0} & 0 \\
0 & D_{d} \\
0 & 0 \\
0 & 0
\end{array}\right]\left[\begin{array}{l}
\zeta(t) \\
\omega(t)
\end{array}\right]+\left[\begin{array}{ll}
G_{0} & 0 \\
0 & 0 \\
0 & B_{p} \\
0 & 0
\end{array}\right]\left[\begin{array}{c}
d_{0 d}(t) \\
(r(t)-d(t))
\end{array}\right] \\
y(t)=d(t)+C_{t} x(t)+E_{t} u_{0}(t-k)
\end{gathered}
$$

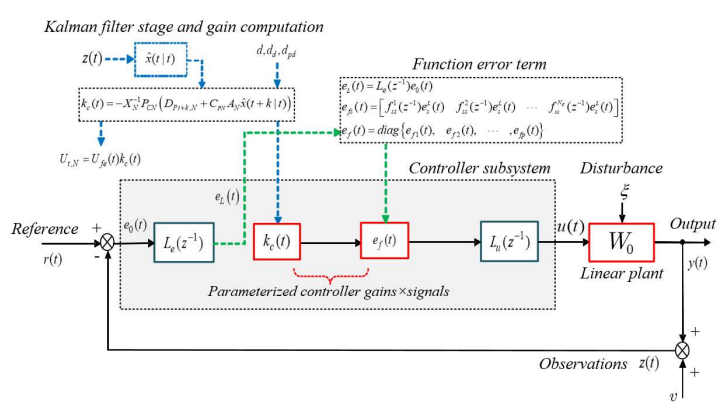

Figure 2. GPFC Control Block Diagram

$$
z(t)=d(t)+C_{t} x(t)+E_{t} u_{0}(t-k)+v(t)
$$

The weighted error to be minimized:

$$
e_{p}(t)=d_{p}(t)+C_{p t} x(t)+E_{p t} u_{0}(t-k)
$$

where $A_{t}, B_{t}, D_{t}, C_{t}, E_{t}, C_{p t}, E_{p t}$ are constant matrices. The prediction of states, at time $t$, is found by repetitive usage of the state in (2) after altering the time using the $k$-steps of explicit transport delay as follow:

$$
\begin{aligned}
& x(t+i+k)=A_{t}^{i} x(t+k)+\sum_{j=1}^{i} A_{t}^{i-j} B_{t} u_{0}(t+j-1) \\
& +D_{t} \xi(t+j+k-1)+d_{d}(t+j+k-1)
\end{aligned}
$$

Similarly, weighted error $e_{p}(t)$ under control prediction has the formulation:

$$
\begin{aligned}
& e_{p}(t+i+k)=d_{p}(t+i+k)+C_{p t} A_{t}^{i} x(t+k) \\
& +\sum_{j=1}^{i} C_{p t} A_{t}^{i-j} B_{t} u_{0}(t+i-1)+D_{t} \xi(t+j+k-1) \\
& +d_{d}(t+j+k-1)+E_{p t} u_{0}(t+i)
\end{aligned}
$$

The $k$-steps ahead tracking error $E_{P_{t+k, N}}$, may consequently be described in following vector arrangement as:

$$
\begin{aligned}
& E_{P t+k, N}=D_{P_{t+k, N}}+C_{p t_{N}} A_{t_{N}} x(t+k) \\
& +V_{P N} U_{t, N}^{0}+C_{p t_{N}} D_{t_{N}} W_{t+k, N}
\end{aligned}
$$

where $V_{P N}=C_{p t_{N}} B_{t_{N}}+E_{p t_{N}}$, and $D_{P_{t+k, N}}$ collects, deterministic disturbance terms in (7). The $i$-steps ahead prediction of error signal, can be produced assuming future actions of the control are identified.

$$
\hat{E}_{P_{t+k, N}}=D_{P_{t+k, N}}+C_{p t_{N}} A_{t_{N}} \hat{x}(t+k)+V_{P N} U_{t, N}^{0}
$$

The state estimation $\hat{x}(t+k \mid t)$, can be calculated $k$-steps ahead in a feasible ordered way via a Kalman filter [10].

\section{LINEAR GPFC}

A brief of a GPFC controller design method for discrete-time linear systems is presented in this section, where for this paper and for the application used, the unstructured first subsystem part is ignored by assuming $W_{l k}=I$. The GPC performance index that inspires the GPFC principle to be minimized with dynamic error weighting, can be defined as in (10).

$$
J=E \sum_{j=0}^{N}\left\{\begin{array}{c}
e_{p}(t+j+k)^{T} e_{p}(t+j+k) \\
+\lambda_{j}^{2}\left[u_{0}(t+j)^{T} u_{0}(t+j)\right]
\end{array}\right\}
$$

where $\lambda_{j}$ represents a scalar control signal weighting. NB, $e_{p}$ is the dynamically weighted error signal and the control weightings can be chosen to be null at later times to provide separate cost horizon's if required. The GPFC cost-function presented here can be defined to have the same form but with a slight enhancement by adding a term to bound the controller gains (denoted $k_{c}$ ) into the cost-index, so that high gain is penalized: 


$$
J=E\left\{J_{t}\right\}=E\left\{\begin{array}{c}
E_{P_{t+k, N}}^{T} E_{P_{t+k, N}} \\
+U_{t, N}^{0 T} \Lambda_{N}^{2} U_{t, N}^{0}+k_{c}^{T} \Lambda_{k}^{2} k_{c} \mid t
\end{array}\right\}
$$

And the cost weightings on future actions $u_{0}$ and controller gains are arranged respectively as:

$$
\begin{aligned}
& \Lambda_{N}^{2}=\operatorname{diag}\left\{\lambda_{0}^{2}, \lambda_{1}^{2}, \cdots, \lambda_{N}^{2}\right\} \\
& \Lambda_{k}^{2}=\operatorname{diag}\left\{\rho_{0}^{2}, \rho_{1}^{2}, \cdots, \rho_{N}^{2}\right\}
\end{aligned}
$$

\section{A. Parameterizing the Predictive Functional Controller}

Before performing the optimization to calculate the optimal control, the controller structure will be constructed to have a chosen, possibly low-order, dynamic form. This is different to model based unconstrained predictive control solutions, where the vector of predicted control actions is calculated and the control at time $t$ is implemented. A restricted structure controller is defined where only gains are to be determined. Hence, all $N_{e}$ linear dynamic functions are selected to have distinct separate frequency response features. Thus, the control signal offered as:

$$
u(t)=L_{u}\left(z^{-1}\right) \sum_{j=1}^{N_{e}} f_{i}\left(z^{-1}\right) k_{j}(t) e_{L}(t)
$$

whereas weighted error $e_{L}(t)=L_{e}\left(z^{-1}\right) e_{0}(t)$ represents a frequency weighting on the error and $L_{u}\left(z^{-1}\right)$ defines a frequency weighting on the system inputs. It may not be necessary and may be just fixed at the identity. However, for multivariable systems where certain loop shapes are required for sensitivity minimization, they may be chosen as in [11] loop-shaping design procedure. This controller structure includes the summation of vector functions which all together will produces the control action as in:

$$
\begin{aligned}
& u(t)= \\
& L_{u}\left(z^{-1}\right)\left\{\begin{array}{l}
f_{1}\left(z^{-1}\right) k_{1} e_{L}(t)+f_{2}\left(z^{-1}\right) k_{2} e_{L}(t) \\
+\cdots+f_{N e}\left(z^{-1}\right) k_{N e} e_{L}(t)
\end{array}\right\}
\end{aligned}
$$

In a simple case, these could be a summation of proportional and integral elements in each channel, e.g., $f_{1}\left(z^{-1}\right) k_{1} e_{L}(t)$ might be proportional term and $f_{2}\left(z^{-1}\right) k_{2} e_{L}(t)$ might represent integral term in each channel. Hence for multivariable form, each of the terms in the summation in the control in (13), $f_{i}\left(z^{-1}\right) k_{i} e_{L}(t)$ are defined to have the next matrix arrangements for each channel $p$ :

$$
\begin{aligned}
& f_{j}\left(z^{-1}\right)=\operatorname{diag}\left\{f_{11}^{j}\left(z^{-1}\right), f_{22}^{j}\left(z^{-1}\right), \cdots, f_{p p}^{j}\left(z^{-1}\right)\right\} \\
& k_{j}=\operatorname{diag}\left\{k_{11}^{j}, k_{22}^{j}, \cdots, k_{p p}^{j}\right\} \\
& e_{L}^{T}(t)=\left[\begin{array}{llll}
e_{1}^{T}(t) & e_{2}^{T}(t) & \cdots & e_{p}^{T}(t)
\end{array}\right]
\end{aligned}
$$

Thence, the $j^{\text {th }}$ functional term for all the channels, multiplied by the error input, introduced as in (16).

$$
f_{j}\left(z^{-1}\right) k_{j} e_{L}(t)=\left[\begin{array}{c}
f_{11}^{j}\left(z^{-1}\right) k_{11}^{j} e_{1}^{L}(t) \\
f_{22}^{j}\left(z^{-1}\right) k_{22}^{j} e_{2}^{L}(t) \\
\vdots \\
f_{p p}^{j}\left(z^{-1}\right) k_{p p}^{j} e_{p}^{L}(t)
\end{array}\right]
$$

Expression (13) offers an accepted parameterization of the controller but, a more suitable form is needed for the optimization of the gains therefore a matrix representation is needed to enable the gains to be collected together in one vector that may then be computed, observing that the function-vector signal for row $s \in[1, p]$, may be defined as:

$$
\begin{aligned}
& e_{f s}(t)= \\
& {\left[f_{s s}^{1}\left(z^{-1}\right) e_{s}^{L}(t) \quad f_{s s}^{2}\left(z^{-1}\right) e_{s}^{L}(t) \quad \cdots \quad f_{s s}^{N_{e}}\left(z^{-1}\right) e_{s}^{L}(t)\right]}
\end{aligned}
$$

Also, the gain set for the $s^{\text {th }}$ channel may be defined as:

$$
k_{c s}=\left[\begin{array}{c}
k_{s s}^{1} \\
k_{s s}^{2} \\
\vdots \\
k_{s s}^{N_{e}}
\end{array}\right]
$$

The $s^{\text {th }}$ row term in (13) may therefore described as:

$$
\sum_{j=1}^{N_{e}} f_{s s}^{j}\left(z^{-1}\right) e_{s}^{L} k_{c s}^{j}(t)=e_{f s}(t) k_{c s}
$$

This corresponds to the vector of scalar gains multiplying each function in the channel $s$. These signals and the gains may be collected together in larger augmented matrices and the total error vector will be a diagonal matrix of the signals defined in (17) will be collected as:

$$
e_{f}(t)=\operatorname{diag}\left[e_{f 1}(t) \quad e_{f 2}(t) \quad \cdots \quad e_{f p}(t)\right]
$$

\section{B. Parameterizing the Vector of Future Controls}

The calculation of the controller gains, utilizing a GPC idea, delivers the gains in (18) in a simple way. Thus, the vector of future actions $U_{t, N}$ is described as:

$$
U_{t, N}=\left[\begin{array}{c}
u(t) \\
u(t+1) \\
\vdots \\
u(t+N
\end{array}\right]=\left[\begin{array}{c}
L_{u}\left(z^{-1}\right) e_{f}(t) \\
L_{u}\left(z^{-1}\right) e_{f}(t+1) \\
\vdots \\
L_{u}\left(z^{-1}\right) e_{f}(t+N)
\end{array}\right] k_{c}(t)
$$

The matrix in (21) assigned $U_{f e}$ and given as:

$$
U_{f e}=\left[\begin{array}{c}
L_{u}\left(z^{-1}\right) e_{f}(t) \\
L_{u}\left(z^{-1}\right) e_{f}(t+1) \\
\vdots \\
L_{u}\left(z^{-1}\right) e_{f}(t+N)
\end{array}\right]
$$

And matrix has $(N+1) \times m$ rows and $p \times N_{e}$ columns. Recalling a GPC action at time $t$ is built on the receding horizon concept [12], the equivalent assumption employed here for GPFC control is that $k_{c}$ can be expected to be remain unchanged during the period $[0, N]$ and the 
calculated $k_{c}$ at time $t$ will be employed to calculate time $t$ optimal control action. And at the next sample time instant, the procedure will start again and a new value of the $k_{c}(t)$ can be calculated following the principal of receding control.

\section{OPTIMIZATION OF THE LINEAR GPFC}

Using the above parameterization of the controller, the cost-function (11) becomes:

$$
\begin{aligned}
& J=\tilde{D}_{P_{t+k, N}^{T}}^{T} \tilde{D}_{P_{t+k, N}}+k_{c}^{T} U_{f e}^{T} V_{P N}^{T} \tilde{D}_{P_{t+k, N}}+\tilde{D}_{P_{t+k, N}}^{T} V_{P N} U_{f e} k_{c} \\
& +k_{c}^{T}\left(U_{f e}^{T}\left(V_{P N}^{T} V_{P N}+\Lambda_{N}^{2}\right) U_{f e}+\Lambda_{K}^{2}\right) k_{c}+J_{0}
\end{aligned}
$$

where

$$
\begin{gathered}
\tilde{D}_{P_{t+k, N}}=D_{P_{t+k, N}}+C_{p t_{N}} A_{t_{N}} \hat{x}(t+k) \\
J_{0}(t)=E\left\{\tilde{E}_{P_{t+k, N}^{T}}^{T} \tilde{E}_{P_{t+k, N}} \mid t\right\}
\end{gathered}
$$

Along with the cost term (25) and $\tilde{E}_{P_{t+k, N}}$ is unassociated to the control action and introduced by the $k$-steps ahead state estimation error in (26)

$$
\begin{aligned}
& \tilde{E}_{P t+k, N}=E_{P t+k, N}-\hat{E}_{P t+k, N} \\
& \tilde{x}(t+k \mid t)=x(t+k \mid t)-\hat{x}(t+k \mid t)
\end{aligned}
$$

Finally, setting the gradient of the cost function to zero, to acquire vector of future optimal control as in [10]. Noting, $J_{0}$ doesn't depends on the control action, then the vector of control gain.

$$
k_{c}(t)=-\left(U_{f e}^{T}\left(V_{P N}^{T} V_{P N}+\Lambda_{N}^{2}\right) U_{f e}+\Lambda_{K}^{2}\right)^{-1} U_{f e}^{T} V_{P N}^{T} \tilde{D}_{P_{t+k, N}}
$$

And the controller can be realized as shown in Fig. 2.

\section{SimUlation AND PERFORMANCE ANALYSIS}

Diagram of QTP is presented in Fig. 3, has been described in [13]. There are two interesting properties about this model. First, this model has large interactions between the tanks 1 and 3 and among tank 2 and tank 4. This is because input from pump 1 fills tank 1 and 4 and in addition the output from tank 3 fills tank 1. Correspondingly, this interaction occurs between tank 2 and tank 4. This interaction has high impact on proper control. Another interesting property, the linearized model of the QTP has a transmission zero, which can be in both left or right half plane by just varying a valve and the system becomes minimum phase or non-minimum phase.

The mathematical equations for QTP using mass balances and Bernoulli's law are:

$$
\begin{aligned}
& \frac{d h_{1}}{d t}=-\frac{a_{1}}{A_{1}} \sqrt{2 g h_{1}}+\frac{a_{3}}{A_{1}} \sqrt{2 g h_{3}}+\frac{\gamma_{1} k_{1}}{A_{1}} v_{1} \\
& \frac{d h_{2}}{d t}=-\frac{a_{2}}{A_{2}} \sqrt{2 g h_{2}}+\frac{a_{4}}{A_{2}} \sqrt{2 g h_{4}}+\frac{\gamma_{2} k_{2}}{A_{2}} v_{2} \\
& \frac{d h_{3}}{d t}=-\frac{a_{3}}{A_{3}} \sqrt{2 g h_{3}}+\frac{\left(1-\gamma_{2}\right) k_{2}}{A_{3}} v_{2} \\
& \frac{d h_{4}}{d t}=-\frac{a_{4}}{A_{4}} \sqrt{2 g h_{4}}+\frac{\left(1-\gamma_{1}\right) k_{1}}{A_{4}} v_{1}
\end{aligned}
$$

\begin{tabular}{cl|c}
\multicolumn{2}{c|}{ Parameter } & Value \\
\hline$A_{1}, A_{3}$ & {$\left[\mathrm{~cm}^{2}\right]$} & 28 \\
$A_{2}, A_{4}$ & {$\left[\mathrm{~cm}^{2}\right]$} & 32 \\
$a_{1}, a_{3}$ & {$\left[\mathrm{~cm}^{2}\right]$} & 0.071 \\
$a_{2}, a_{4}$ & {$\left[\mathrm{~cm}^{2}\right]$} & 0.057 \\
$k_{c}$ & {$[\mathrm{~V} / \mathrm{cm}]$} & 0.5 \\
$G$ & {$\left[\mathrm{~cm} / \mathrm{s}^{2}\right]$} & 981
\end{tabular}

Given $\gamma_{l}$ is the flow spreading to lower and diagonal upper tank, $A_{l}$ is the cross-section area, $a_{l}$ is the drain hole crosssection and $h_{l}$ is liquid level, in tank $l$ correspondingly. QTP have two inputs and two outputs and control objective is to keep liquid level in lower tanks close to its setpoint by controlling liquid flow by the attached two pumps. The voltage that control these pumps are $v_{1}$ and $v_{2}$, the corresponding flow is $k_{l} v_{l}$ and the outputs are $y_{l}=k_{c} h_{l}$ and $y_{2}=k_{c} h_{2}, k_{c}$ is the level sensor parameter. The parameter values of the QTP are specified in Table I. The parameters $\gamma_{1}, \gamma_{2} \in(0,1)$ are valves setting for the spreading of flow to lower and upper diagonal tank correspondingly. The flow to Tank 1 is $\gamma_{1} k_{1} v_{l}$ and is $\left(1-\gamma_{1}\right) k_{l} v_{1}$ to Tank 4 and also for Tank 2 and Tank 3 as in Fig. 3. The $g$ is the acceleration of gravity. This system has two finite zeros for $\gamma_{1}, \gamma_{2} \in(0,1)$. One continually lies in the left half plane, but the other could be chosen to be in the left or right half plane based on the valve values of $\gamma_{1}, \gamma_{2}$ as follow:

- $1<\gamma_{1}+\gamma_{2} \leq 2$ QTP is minimum phase.

- $1 \leq \gamma_{1}+\gamma_{2}<1$ QTP is non-minimum phase.

- $\gamma_{1}+\gamma_{2}=1 \quad$ QTP has transmission zero at origin.

The control of the QTP are considered for both operating points, $P^{-}$when the system will have minimum phase properties and $P^{+}$when it will have nonminimum phase properties. The chosen operating points linked to the parameter values are shown in Table II.

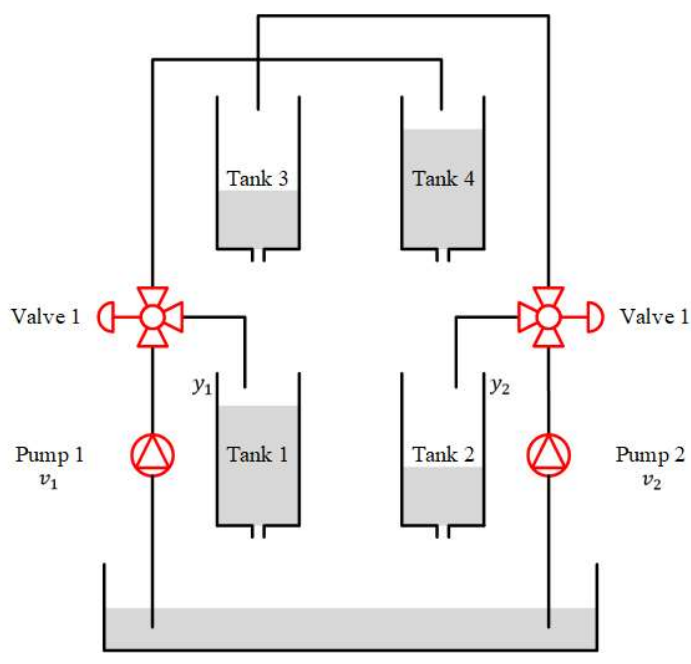

Figure 3. Illustrative drawing of the QTP 
Introduce these variables:

$$
\begin{aligned}
& x_{i}=h_{i}-h_{i}^{0} \\
& u_{i}=v_{i}-v_{i}^{0}
\end{aligned}
$$

The linearized state space equation and the multi-loop PI tuning parameters are found in [13], where $K_{p l}, T_{i l}=3.0$, $30, K_{p 2}, T_{i 2}=2.7,40$ for minimum phase and $K_{p 1}, T_{i 1}=1.5$, $110, K_{p 2}, T_{i 2}=-0.12,220$ for non-minimum phase process correspondingly.

After simple multi loop PI control been utilized for both cases, an overshoot has been observed at 7.3 with settling time about 60 seconds as depicted in Fig. 4 (Up) in the minimum phase case. And in the non-minimum phase case, a small inverse response combined with increased overshoot at 8.2 with settling time about 1942 seconds has been observed in the simulation as shown in Fig. 4 (Down), the results are slightly different from [13], since simulation have been implemented in time domain using discretized plant via state space approach in comparison with Johansson, frequency domain method [14].

To complete the assessment, firstly a restricted structure PI controller has been applied to control the nonlinear quadruple tank problem model as a regulating problem to assist the controller regulating futures. For the minimum phase case, the prediction horizon was chosen as $N=40$, and the control horizon as $N_{u}=40$ and observed response of $Y_{l}$ and $Y_{2}$ with no overshot and settling time i.e., 25 seconds as depicted in Fig. 4 (Up). Also, for the non-minimum phase case, prediction horizon $N=80$, and control horizon $N_{u}=20$ has been used and simulation shows, an inverse response along with smaller overshoot at 7.6 for 6.5 set point with settling time around 600 seconds as shown in Fig. 4 (Down). Secondly, to assist the GPFC tracking future, a restricted structure PID controller has been applied to
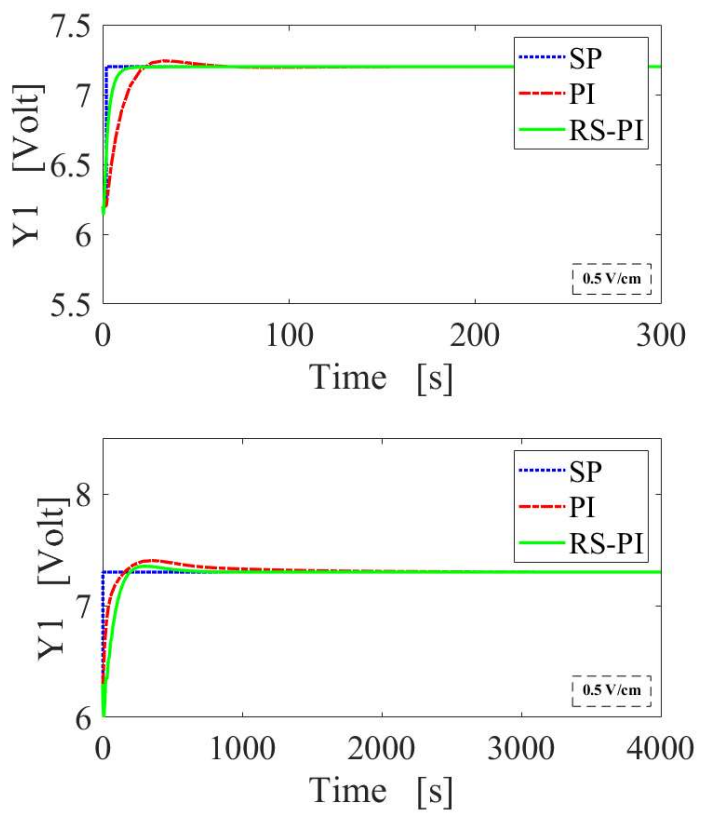

TABLE II. OPERATING PARAMETER, P- AND P+ PHASES

\begin{tabular}{cc|c|c} 
& & $\boldsymbol{P}$ - & $\boldsymbol{P}+$ \\
\hline$h_{1}^{0}, h_{2}^{0}$ & {$[\mathrm{~cm}]$} & $(12.4,12.7)$ & $(12.6,13.0)$ \\
$h_{3}^{0}, h_{4}^{0}$ & {$[\mathrm{~cm}]$} & $(1.8,1.4)$ & $(4.8,4.9)$ \\
$v_{1}^{0}, v_{2}^{0}$ & {$[V]$} & $(3.00,3.00)$ & $(3.15,3.15)$ \\
$k_{1}, k_{2}$ & {$\left[\mathrm{~cm}^{3} / V_{s}\right]$} & $(3.33,3.35)$ & $(3.14,3.29)$ \\
$\gamma_{1}, \gamma_{2}$ & & $(0.70,0.60)$ & $(0.43,0.34)$
\end{tabular}

control the nonlinear quadruple tank model to track the change in the set point and to reject the output disturbance in a MIMO system with high interaction between the different loops as shown in Fig. 5. A step disturbance has been applied to the process outputs at different times and the simulation shows a good controller performance in rejecting these disturbance variables whilst at the same time the controller maintains good tracking to changes in different setpoints.

\section{CONCLUSION}

In this paper, a MIMO GPFC controller has been designed based on linearized model to control a nonlinear model of quadruple tank and been simulated in both structures of a PI and PID design and was employed in both regulating and tracking problems, where the optimized time varying controller gains, are continuously adjusted. In this future knowledge, GPFC control adjust to the variations in reference trajectories and disturbance rejection. Performance assessment of the controller to that of a classical PI and PID controllers has been completed and the outcomes demonstrate improved performance. GPFC controller could easily be extended to control different classes of nonlinear systems i.e. quasi-LPV systems.
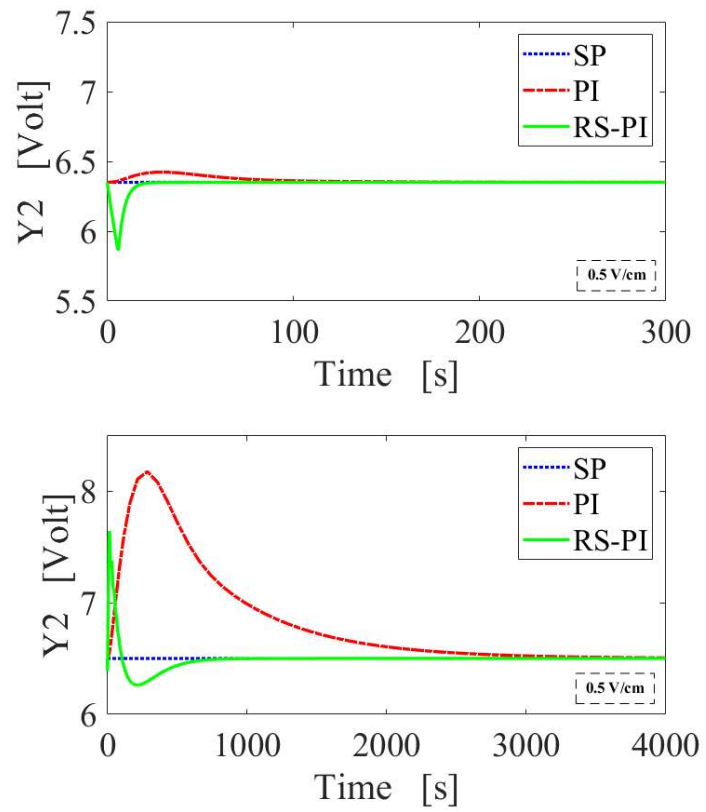

Figure 4. GPFC-PI vs Classic PI Controller Performance, Minimum Phase (Up), Non-minimum Phase (Down) 

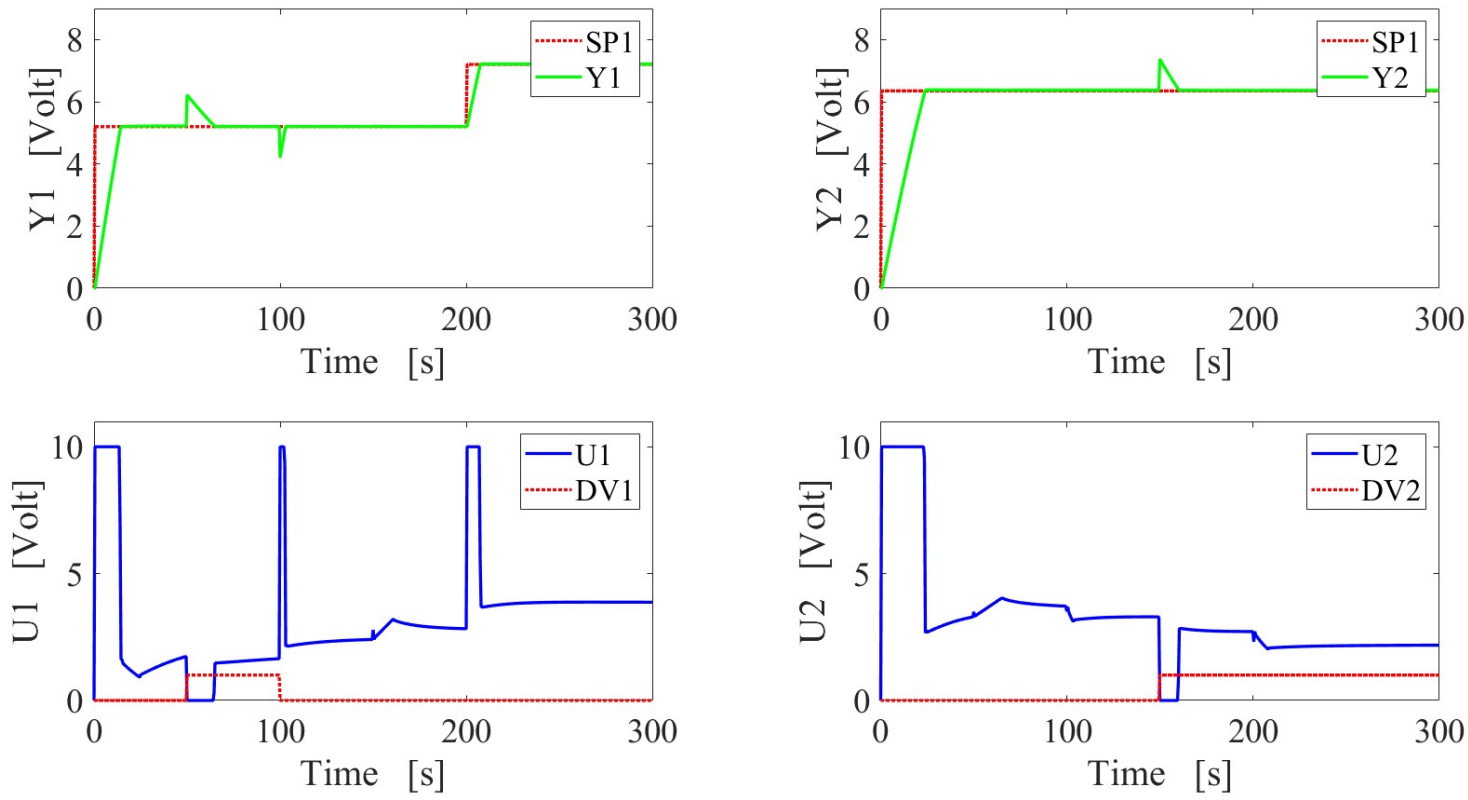

Figure 5. GPFC-PID Reference Tracking and Disturbance Rejection Performance

\section{ACKNOWLEDGMENT}

The author S. Alotaibi would like to express his gratitude and thanks Dr Pawel Majecki, ISC Ltd. for his support on NPGMV toolbox software

\section{REFERENCES}

[1] R. M. Miller, S. L. Shah, R. K. Wood, and E. K. Kwok, "Predictive PID," ISA Trans., vol. 38, no. 1, pp. 11-23, Jan. 1999.

[2] J. Richalet, "Industrial applications of model based predictive control," Automatica, vol. 29, no. 5, pp. 1251-1274, 1993.

[3] J. Richalet, "Commande prédictive," Autom. ingénierie système, R7423, pp. 1-17, Dec. 1997.

[4] J. A. Rossiter and J. Richalet, "Handling constraints with predictive functional control of unstable processes," Proc. Am. Control Conf., vol. 6, pp. 4746-4751, 2002.

[5] M. H. Moradi, M. R. Katebi, and M. A. Johnson, "Predictive PID control: a new algorithm," vol. 1. USA, pp. 764-769, 2001.

[6] D. Uduehi, D. Ordys, D. Grimble, A. Ordys, and M. J. Grimble, "Multivariable PID controller deign using online generalised predictive control optimisation," vol. 1. USA, pp. 272-277, 2002.

[7] M. J. Grimble and P. Majecki, "State- space approach to nonlinear predictive generalised minimum variance control," Int. J. Control, vol. 83, no. 8, pp. 1529-1547, 2010.

[8] M. J. M. J. Grimble and P. Majecki, "Restricted structure predictive control for linear and nonlinear systems," Int. J. Control, pp. 1-61, Oct. 2018.

[9] M. J. Grimble, Industrial control systems design. Wiley, 2001.

[10] M. J. Grimble and M. A. Johnson, Optimal control and stochastic estimation : theory and applications. Wiley, 1988.

[11] D. C. McFarlane and K. Glover, Robust Controller Design Using Normalized Coprime Factor Plant Descriptions, vol. 138 Berlin/Heidelberg: Springer-Verlag, 1990.

[12] W. Kwon and A. Pearson, "A modified quadratic cost problem and feedback stabilization of a linear system," IEEE Trans. Automat. Contr., vol. 22, no. 5, Oct. 1977.

[13] K. H. Johansson, "The quadruple-tank process: a multivariable laboratory process with an adjustable zero," IEEE Trans. Control Syst. Technol., vol. 8, no. 3, pp. 456-465, May 2000.
[14] Q. Saeed, V. Uddin, and R. Katebi, "MIMO Predictive PID Control: A Practical Approach For Quadruple Tank," J. Circuits, Syst. Comput., vol. 22, no. 03, p. 1350009, Mar. 2013. 